\title{
Hospital nurses' attitudes, negative perceptions, and negative acts regarding workplace bullying
}

\author{
Shu-Ching Ma ${ }^{1,2,5}$, Hsiu-Hung Wang ${ }^{1}$ and Tsair-Wei Chien ${ }^{3,4^{*}}$ (i)
}

\begin{abstract}
Background: Workplace bullying is a prevalent problem in today's work places that has adverse effects on both bullying victims and organizations. To investigate the predictors of workplace bullying is an important task to prevent bullying victims of nurses in hospitals.

Objective: This study aims to explore the relationships among nurses' attitudes, negative perceptions, and negative acts regarding workplace bullying under the framework of the theory of planned behavior (TPB).

Methods: A total of 811 nurses from three hospitals in Taiwan were surveyed. Nurses' responses to the 201 items of 10 scales were calibrated using Rasch analysis and then subjected to path analysis with partial least-squares structural equation modeling (PLS-SEM).

Results: The instrumental attitude was significant predictors of nurses' negative perceptions to be bullied in the workplace. Instead, the other TPB components of subjective norm and perceived behavioral control were not effective predictors of nurses' negative acts regarding workplace bullying.

Conclusions: The findings provided hospital nurse management with important implications for prevention of bullying, particularly to them who are tasked with providing safer and more productive workplaces to hospital nurses. Awareness of workplace bullying was recommended to other kinds of workplaces for further studies in future.
\end{abstract}

Keywords: Workplace bullying, Theory of planned behavior, Rasch measurement, Path analysis, Partial least squares structural equation modeling

\section{Introduction}

Workplace bullying occurs when an employee experiences a persistent pattern of mistreatment from others in the workplace that causes harm [1]. Likely, workplace bullying is persistent exposure to interpersonal aggression and mistreatment from colleagues, superiors, or subordinates [2,3]. The form of bullying can include such expressions as verbal, nonverbal, psychological, physical abuse, humiliation and cyber [4]. Unlike the forms of school bullying in the workplace bullying, workplace bullying is in the majority of cases reported as having been

\footnotetext{
${ }^{*}$ Correspondence: smile@mail.chimei.org.tw

${ }^{3}$ Research Department, Chi-Mei Medical Center, 901 Chung Hwa Road, Yung Kung Dist., Tainan 710, Taiwan

Full list of author information is available at the end of the article
}

perpetrated by someone in authority over the target, sometimes from peers, and occasionally from subordinates [5].

Bullying can be covert or overt. It is frequently missed by superiors and well known by many employees throughout the organization. Researchers have done impressive studies investigating this problem by determining its frequency, identifying groups at risk in different occupational groups and sectors [6], addressing prevalence of bullying in different countries and among different occupational groups [7], reporting the impact on bullying in a workplace and the group-level processes that impact on the incidence and maintenance of bullying behavior [8], detecting the appropriateness of level of the bully scaling [9], testing a multidimensional model of 
bullying in the nursing workplace [10], and even exploring a computer adaptive testing (CAT) tactic to examine hospital nurses' perception of workplace bullying [11]. However, they all have focused on only one aspect of assessment regarding bully attitudes or negative acts, or merely on a single correlation between attitudes and negative acts but failed to investigate the correlation between these variables under a sound theoretical framework, for instance, using theory of planned behavior (TPB) [12] to examine the relations of those variable domains.

\section{PLS-SEM used for exploring the relationships between these variables}

The counterproductively negative effects like bully are not limited to the targeted individuals but led to a decline in employee morale and a change in an organizational culture. None to date was to fill this gap through a comprehensively overall viewpoint by exploring the relationships among nurses' attitudes, negative perceptions, and negative acts regarding workplace bullying under the framework of the TPB, particularly, using the method of partial least squares structural equation modeling (PLSSEM). The PLS-SEM is evolving as a statistical modeling technique and its use has increased exponentially in recent years within a variety of disciplines, due to the recognition that PLS-SEM's distinctive methodological features make it a viable alternative to the more popular covariance-based SEM approach [13] in social sciences.

\section{Theory of planned behavior applied to this study}

The TPB proposed by Ajzen $[12,14]$ is a rigorous theoretical framework to provide prediction and explanation of examinees' intentions to behavior. TPB has been successfully applied to provide a better interpretation of diverse behaviors in western settings [15]. According to TPB theory, three determinants-including attitude (i.e., whether I want to or not to support something), subjective norms (i.e., whether others encourage or limit me to support or not to support something), and perceived behavioral control (i.e., whether I have opportunities and resources to do or not to do something)-exert their effects on behavior through intentions [12] presented in Fig. 1.

In the current study, we applied the counterproductive TPB (CP-TPB for short in this study) concept to the bully negative acts through its underlying negative perceptions predicted by the other three determinants (i.e., attitude, subjective norms, and perceived behavioral control).

Attitude is denoted as the personal orientation with a positive or negative thinking. The orientation often contains two components: (1) affective and (2) instrumental attitude $[12,16]$. The affective attitude is related to feelings or emotions positively or negatively toward a target; while instrumental attitude carries an appraisal

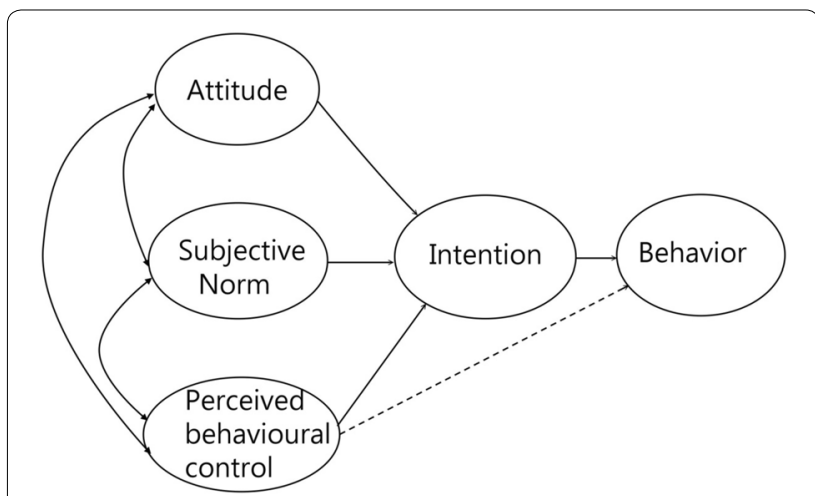

Fig. 1 The model of theory of planned behavior

of the consequences of the target. As such, a paper [17] reported that the negative Automatic Thoughts Questionnaires (ATQ) score [18] was positively correlated with the Liebowitz Social Anxiety Scale (LSAS) scores and the positive ATQ score was negatively correlated with the LSAS scores [17].

Subjective norm refers to perceived social pressure from others to perform (or not perform) the behavior. Perceived behavioral control refers to one's perception of the ability and control over the target. Self-efficacy is a perceived ability or a controllability, which refers to people's beliefs that they have the ability to resist any negative acts such as the bully behavior.

Negative acts might be perceived by a series of negative feelings such as poor mental health, burnout, and intention to resign. That is, one holding a negative attitude is possible to yield negative perceptions that might lead to a feeling of all negative acts around him or her. For more information about the definition of each study scale, we provide a couple of practical examples (e.g., original questions) on their specific meaning and implication in Additional file 1.

Generally, those with a favorable attitude, positive subjective norms, and a high level of perceived behavioral control (i.e., self-efficacy) will more likely earn a low probability of negative perceptions and negative acts. Similarly, a low degree of organizational subject norm and personal perceived behavioral control possibly links to negative acts through the negative perceptions. It is required to replace the TPB from need-to-do-it with the CP-TPB avoidance-to-do-it for interpreting the relationship of components with a study model.

\section{Aims of the study}

This study aims at examining the extent to which CP-TPB can predict and explain nurses' negative perceptions and negative acts of a bullied victim in a hospital. The following two specific hypotheses are tested in Fig. 1: 
(1) Nurses' negative perceptions to negative acts can be predicted by attitude, subjective norm, and perceived behavioral control regarding workplace bullying; and (2) nurses' negative acts can be predicted by negative perceptions and perceived behavioral control regarding workplace bullying.

\section{Methods}

\section{Study participants}

The study sample was randomly selected and recruited using the last 3 digits of the identification card number from nurses of a group of hospitals with 2133 beds in southern Taiwan in the summer of 2012. As an incentive for participation, a gift consumer card for US\$6.40 good for purchases at 7-11 convenience stores was offered to participants. A total of 811 nurses completed 201 items for 10 scales (e.g., each one illustrated by several items, see Additional file 1). This study was approved and monitored by the Research Ethics Review Board of the Chi-Mei Medical Center. Demographic data collected included gender, work tenure in hospitals of all types, age, marital status, and education level.

\section{Instruments}

A Nurse's Conceptions Regarding Workplace Bullying Questionnaire containing 201 item of 10 scales was developed to assess the five components in the TPB framework in Fig. 1. The scales included two parts: (A) negatively inversed scores (the higher scores, the more negative perceptions or acts): workplace bullying, intent to resign, nurse burnout, and personal mental disorder, (B) positively increased monotonically scores (the higher scores, the more positive effects on persons or organizations): job satisfactory, service spirit, authority distance, leadership of nurse superiors, organization culture, and personality. Participants were asked to rate each item on a 5-point scale with response options ranging from strongly disagree (1) to strongly agree (5). A higher score represents a higher level of the respective latent trait of the aforementioned two kinds of negative and positive scales under investigation.

The scale development procedure was guided by $\mathrm{DeV}$ ellis' instruction [19] and item crafting was guided by Ajzen's principles [16] for TPB scale construction. The questions were constructed based on previous literature on workplace bullying [9-11], and consultative discussions with relevant experts (in the field of health care assessment) as well as frontline nurses who have had first-hand experiences in nursing care. The scales were then subjected to a pilot test on a small sample of nurses $(n=32)$ for the purpose of helping refine those questions by identifying ambiguities and anomalies in items of wording, as well as possible bias.
The scores on negatively worded items just in Authority distance scale were reversed before the data analysis so as to maintain the consistency of interpretation. The other three scales of mental disorder, burnout, and intent to resign with negatively worded items are kept with original codes for data analysis.

\section{Data analysis}

Two analytical methods, i.e., Rasch analysis [20] and path analysis, were used in the present study. Rasch rating scale analysis using Winsteps 3.7 [21] was used for examining the psychometric properties of the ten scales and for calibrating nurse' (person) measures on each of the ten latent traits. The Rasch estimated person measures were subsequently analyzed by path analysis using PLS-SEM [13] to investigate the relationships among components under CP-TPB model. This approach to data analysis differs from the conventional SEM method containing all indicators to fulfill the function of measurement model. In contrast, we applied Rasch analysis to PLS-SEM for measuring the latent (unobserved) traits using those ten underlying measures.

An inherent weakness associated with conventional analytical techniques based on classic test theory (CTT), such as factor analysis, is that they require linear, interval scale data input [22]. Raw data collected through Likert-type scales are always ordinal since their categories indicate its ordering without any proportional levels of meaning $[23,24]$. Therefore, it is highly possible misleading conclusions if applying CTT to raw scores which are ordinal data (i.e., response from 1 to 5 ordered category) in nature.

The Rasch model overcomes this problem by converting ordinal data into interval measures which have a constant interval meaning and provide objective measurement from ordered category responses [24]. Once the interval metric is established, person measures and item difficulties are to be calibrated onto a single unidimensional latent trait continuum which facilitates direct comparisons between person measures and item difficulties. Empirically, Rasch analysis has been successfully applied in education and social sciences in addressing assessment issues $[23,25,26]$.

Multiple criteria including Rasch person/item reliability, item fit statistics, the amount of variance explained by each of the scale measures, and step thresholds are used to examine the psychometric properties of those scales. Rasch person/item reliability estimates the replicability of person/item ordering along the latent trait metric [23]. Item fit statistics estimate the extent to which the data matches the measurement specifications of the Rasch model. Outfit and infit mean squares (MNSQ) are widely used indices of item fit statistics. The values of Outfit 
and Infit MNSQ (range from 0 to positive infinity) with 1.0 indicating the (unattainable) perfect fit to the Rasch model. Researchers [27] suggested that MNSQs falling in the range of 0.6 and 1.4 indicated a productive measurement for survey data with rating scales. This criterion (i.e., MNSQ in a range of 0.6 and 1.4) was used as the cut-off value of MNSQ fit statistics in this study. Variance explained by Rasch measures refers to the proportion of variance in the observed data which can be explained by the Rasch measures [25]. A higher proportion of variance indicates that the Rasch model better predicts both items and persons. Step threshold difficulties are examined to ensure the appropriate category functioning of the rating scales by Linacre suggested guidelines [28].

In path analysis, the PLS-SEM method [13, 29] was applied to investigate the correlation between components under the theoretical framework of CP-TPB model. The path coefficients between any two components under the CP-TPB using PLS-SEM were examined by the criterion of type I error at 0.05 level.

\section{Results}

Psychometric properties of the scales

The psychometric properties of the ten scales were investigated from a Rasch measurement perspective. Any item mis-fitting to the Rasch model (both infit and outfit MNSQ being higher than 1.4) was removed from the responding scale with an approach of one at a time (i.e., each run just for one deleted item) according to the misfit order [30], and re-applied Rasch analysis until all remaining items showed sufficient fit to the Rasch model. Table 1 presents the summary of psychometric properties of all scales.

It is shown in Table 1 that the Rasch person/item reliabilities for all scales are higher than 0.80 except the person reliability for the intent to resign and authority distance scales due to a short length of items. Rasch measures explained over $40 \%$ of the variables observed in the data for all scales. The results indicated that all the ten scales had quite good psychometric properties at an acceptable scaling quality. Table 1 presents the summary of psychometric properties of all scales. The final sample items of the scales are illustrated in Additional file 1.

The category functioning of the rating scales were examined to determine whether respondents used all response opportunities appropriately. It can be seen that the step thresholds (the intersection point between consecutive categories) advanced monotonically with the category, indicating that the 5-point rating scale functioned rather well, and meaning higher performance categories corresponded to higher measures of the latent trait. In summary, the results showed that the scales were psychometrically robust enough for use with the sample in the current study.

\section{Descriptive statistics}

Descriptive statistics were undertaken to provide an overall viewpoint of the interval Rasch-calibrated measures of nurses on the ten constructs. Table 2 presents means (in a log odds unit) and standard deviations (SD) of nurses' measures on the scales as well as Pearson correlations among the constructs of interest. In Rasch analysis, the mean of item difficulties is arbitrarily set to zero and the interpretation of item difficulties and person measures are based on pair-wise comparisons between items and persons. Therefore, person measures higher than zero indicate a positive response, while person measures lower than zero that indicate a negative response (e.g., the first four scales with negatively inversed scores present not serious because scores are less than zero).

Table 1 Psychometric properties of measurement scales

\begin{tabular}{|c|c|c|c|c|c|c|c|}
\hline \multirow[t]{2}{*}{ Scale } & \multirow[t]{2}{*}{ No. of items } & \multirow{2}{*}{$\begin{array}{l}\text { Rasch person/item reli- } \\
\text { ability }\end{array}$} & \multirow{2}{*}{$\begin{array}{l}\text { Variance explained } \\
\text { by measures (\%) }\end{array}$} & \multicolumn{4}{|c|}{ Step threshold } \\
\hline & & & & Step 1 & Step 2 & Step 3 & Step 4 \\
\hline \multicolumn{8}{|c|}{ A. The higher scores, the more negative perceptions or acts } \\
\hline Bullying acts & 22 & $0.90 / 0.98$ & 41.90 & -2.30 & -0.13 & 0.44 & 1.99 \\
\hline Intent to resign & 6 & $0.76 / 1.00$ & 51.80 & -1.36 & -0.57 & 0.20 & 1.65 \\
\hline Burnout & 11 & $0.90 / 1.00$ & 61.40 & -3.89 & -1.00 & 1.76 & 3.13 \\
\hline Mental disorder & 6 & $0.83 / 1.00$ & 67.20 & -4.19 & -0.69 & 1.81 & 3.07 \\
\hline \multicolumn{8}{|c|}{ B. The higher scores, the more positive effects on persons or organizations } \\
\hline Job satisfaction & 20 & $0.92 / 0.99$ & 51.30 & -3.77 & -2.52 & 0.89 & 5.39 \\
\hline Service spirit & 17 & $0.92 / 1.00$ & 56.90 & -4.73 & -2.63 & 1.49 & 5.78 \\
\hline Authority distance & 6 & $0.75 / 0.99$ & 43.90 & -2.96 & -0.53 & 0.95 & 2.54 \\
\hline Leadership & 50 & $0.83 / 1.00$ & 42.50 & -2.30 & -0.48 & 0.50 & 2.29 \\
\hline Organization culture & 20 & $0.84 / 0.97$ & 41.00 & -2.32 & -1.00 & 0.42 & 2.00 \\
\hline Personality & 43 & $0.91 / 1.00$ & 44.50 & -2.97 & -1.39 & 0.56 & 3.81 \\
\hline
\end{tabular}


Table 2 Means, standard deviations, and correlations of the study components

\begin{tabular}{|c|c|c|c|c|c|c|c|c|c|c|c|c|}
\hline & Component & Mean & SD & (1) & (2) & (3) & (4) & (5) & (6) & (7) & (8) & (9) \\
\hline (1) & Bullying acts & -4.76 & 1.84 & - & & & & & & & & \\
\hline (2) & Intent to resign & -0.76 & 0.89 & $0.23^{* *}$ & - & & & & & & & \\
\hline (3) & Burnout & -1.64 & 2.08 & $0.26^{* *}$ & $0.23^{* *}$ & - & & & & & & \\
\hline (4) & Mental disorder & -3.54 & 2.64 & $0.34^{* *}$ & $0.14^{* *}$ & $0.47^{* *}$ & - & & & & & \\
\hline (5) & Job satisfaction & 1.36 & 1.88 & $-0.32^{* *}$ & $-0.29^{* *}$ & $-0.39 * *$ & $-0.3^{* *}$ & - & & & & \\
\hline (6) & Service spirit & 3.13 & 2.20 & $-0.18^{* *}$ & $-0.19^{* *}$ & $-0.25^{* *}$ & $-0.12^{* *}$ & $0.61^{* *}$ & - & & & \\
\hline (7) & Authority distance & 1.31 & 1.26 & 0.02 & 0.01 & 0.03 & 0.00 & -0.06 & -0.06 & - & & \\
\hline (8) & Leadership & 1.55 & 0.49 & -0.02 & -0.02 & 0.00 & 0.04 & -0.04 & 0.00 & $0.22^{* *}$ & - & \\
\hline (9) & Organization culture & 0.70 & 1.19 & -0.02 & -0.01 & 0.01 & 0.03 & 0.02 & 0.00 & $-0.07^{*}$ & $0.22^{* *}$ & - \\
\hline (10) & Personality & 2.76 & 0.92 & -0.04 & -0.04 & -0.01 & 0.01 & 0.02 & 0.02 & -0.02 & 0.10 & $0.33^{* *}$ \\
\hline
\end{tabular}

${ }^{*} p<0.05$;** $p<0.01$

It is shown in Table 2 that, in general, nurses held a substantially low level of negative perceptions [i.e., scales (2) $-(4)$ ] to potentially be bullied victims [i.e., the scale (1)] with means less than zero. Attitude, both job satisfaction (mean $=1.36$ ) and service spirit $(=3.13)$, and subject norm with means of 1.31 and 1.55 regarding authority distance and leadership were quite positive, while perceived behavioral controls, i.e., organization culture $($ mean $=0.70)$ and personality (mean $=2.76$ ), were positive.

It is worth noting that all of those respective latent traits were significantly related within the component (i.e., with a good convergent validity) and unrelated between components (i.e., with a good discriminant validity). Nurses had a slightly negative mean measure on bully acts (mean $=-4.76$ ). This indicates that, according to the direct effect from the nearby nurses' negative perceptions and the indirect effect from the three far-left components, a rather good hospital climate was evident and led to a lower negative perceptions and a very low negative acts of workplace bullying.

\section{Results of path analysis}

Nurses Rasch-calibrated measures on the CP-TPB components are subsequently subjected to path analysis, aiming at addressing the main research questions: to explore whether nurses' negative perceptions to negative acts can be predicted by attitude, subjective norm, and perceived behavioral control regarding workplace bullying. The results of path analysis showed that the standardized regression weight of the path $(-0.40)$ from attitude to negative perceptions was significant $(p<0.01)$. The paths from subjective norm $(=-0.006)$ and perceived behavioral control $(=0.004)$ to negative perceptions were not significant. The correlations between the three far-left components were virtually zero. The standardized regression weight of the path from negative perceptions to negative acts $(=0.38)$ was significant $(p<0.01)$, while the paths from perceived behavioral control component $(=-0.03)$ were not significant.
The proposed model accounts for $16.4 \%$ of the variances in nurses' negative perceptions with a statistical power of 0.99 (calculated by 3 predictors, 813 sample size, probability level at 0.05 , and observed $R^{2}=0.164$ ) and accounts for $14.9 \%$ of the variances with a statistical power of 0.99 (computed by 2 predictors, 813 sample size, probability level at 0.05 , and observed $R^{2}=0.149$ ) in nurses' negative acts. The relationships among the latent traits in the CP-TPB are presented in Fig. 2.

The direct and indirect effect coefficients of predictors on workplace bullying are presented in Table 3 . In the proposed model, negative perceptions (via direct effect) had a substantial positive effect $(=0.38)$ on negative acts; attitude predictor (via indirect effect) had a moderate negative effect $(=-0.16)$ on negative acts, while the other predictors had a far less than substantial effect on negative acts.

\section{Discussion}

This study used CP-TPB as a theoretical framework to verify that (1) nurses' negative perceptions to negative acts can be predicted by personal attitude; and (2) nurses'

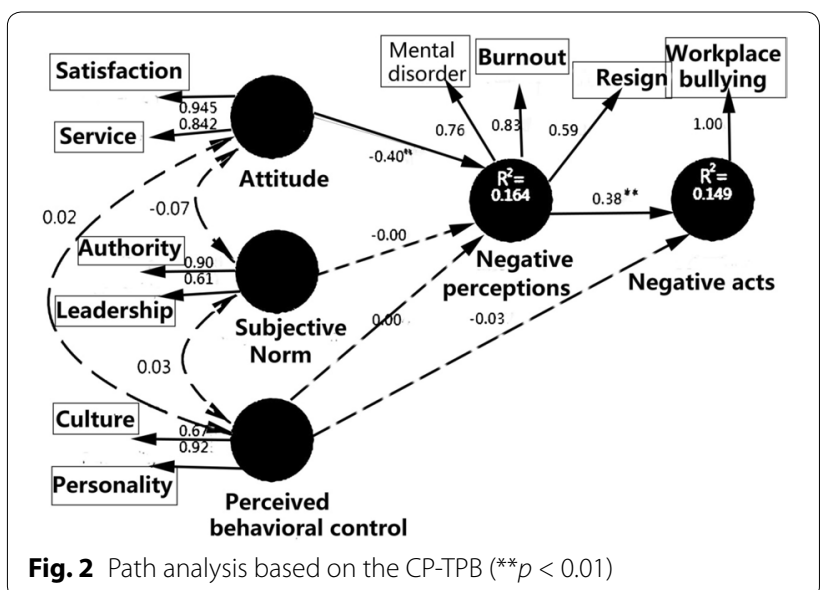


Table 3 Effects of each latent variable on the bully behavior

\begin{tabular}{lllc}
\hline Predictor components & Direct effect & Indirect effect & Total effect \\
\hline Negative perceptions & 0.38 & - & 0.38 \\
Attitude & - & -0.16 & -0.16 \\
Subjective norm & - & -0.01 & -0.01 \\
Perceived behavioral & -0.04 & - & -0.04 \\
$\quad$ control & & & \\
\hline
\end{tabular}

negative acts regarding workplace bullying can be predicted by negative perceptions.

\section{What this adds to what was known}

Many previous pieces of research [5-11] have investigated the impact on bullying in a workplace and the group-level processes that impact on the incidence and maintenance of bullying behavior, but few attempts have been made to build a structural understanding of workplace bullying or of the relationships among variables which have influences on nurses' negative perceptions and negative acts of bullies.

The approach adopted for the analysis was a two-step process with Rasch analysis followed by path analysis. Rasch model can convert ordinal data into interval measures [24] and deal with missing data [31,32] which are problematic in CTT approaches [33-35]. The Rasch person reliabilities for the intent to resign and authority distance scales are less than 0.80 in Table 1 . It is because a shorter length of six items.

In path analysis, the PLS-SEM method has recently gained increasing attention, especially for the management information systems [36], as well as in marketing [37] and strategic management [38] disciplines, but also in accounting [39], family business research [40], operations management [41], and in organizational research [42]. The method is currently regarded as suitable and, to some extent, a favorable alternative to the more restrictive traditionally used covariance-based SEM (CB-SEM) method [43].

Among nurses, the prevalence of bullying was reported to be widespread [44, 45], with estimates suggesting $80 \%$ of nurses experience bullying at some point in their working lives [46]. The consequences of bullying include the following: severe psychological trauma [47]; lowered self-esteem [48]; depression and anxiety [49]; post-traumatic stress disorder [50]; physical illness [51]; financial loss; and the eventual inability to work [52]. The ripple effect of bullying also extends to family members who are liable to experience considerable stress from living with a family member who has been bullied [53]. The findings of this study can provide hospital nurse management with important implications for prevention of bullying, particularly to them who are tasked with providing safer and more productive workplaces to hospital nurses.

\section{What it implies and what should be changed?}

The explanatory model of bullying resulting from this study in Fig. 2 identifies three components (low attitude $\rightarrow$ negative perceptions $\rightarrow$ negative acts) relations that contribute to bullying features, the relationship between bullying acts and the antecedent predictors. The model provides an insight into developing additional strategies to manage workplace bullying. In particular, the model may assist nurse managers to understand features of the work climate that perpetuate the bully behavior. Importantly, the model draws attention to personal attitude (i.e., satisfying nurse job and enjoying healthcare service) that contribute to reduce personal negative perceptions and negative acts regarding workplace bullying.

\section{Strengths of this study}

We applied Rasch model to detect data unidimensional, to establish interval metric for SEM modeling, and to interpret the relationship between components of latent traits. Empirically, Rasch analysis has been successfully applied in education and social sciences in addressing assessment issues $[23,25,26]$ and worth applying to this study.

The Rasch estimated person measures were subsequently analyzed by path analysis using PLS-SEM [13] to investigate the relationships among components under CP-TPB model. The approach differs from the conventional SEM method containing all indicators to fulfill the function of the measurement model. We, on the other hands, applied Rasch analysis to PLS-SEM for measuring the latent (unobserved) traits using those ten underlying measures.

Structural equation modeling (SEM) has become the methodology of choice for many social science researchers investigating complex relationships between latent constructs, such as those ten components in this study. Compared with another commonly used approach of CBSEM subjected to data normal distribution assumptions and to have a not-too-small sample size, there are many advantages in applying PLS-SEM [43].

\section{Definitions of being bullied and engaging in bullying}

This study sample was drawn from those frontline nurses who have had first-hand experiences in nursing care. All questions of the 22-item Negative Acts QuestionnaireRevised (NAQ-R) questionnaire [54] are pertaining to frequency of being bullied. We adopted the definition of bullying [55]: when a person is teased repeatedly in a way he or she does not like... But it is not bullying when two students of about the same strength or power argue or 
fight. It is also not bullying when a student is teased in a friendly and playful way.

Olweus [56] identified three prominent characteristics of bullying behavior: negative actions, repetition, and power imbalance. The negative perceptions were thus rated by victims of bullying instead of those nurses who engage in bullying behavior. Otherwise, those scale quality indices shown in Table 1 and Fig. 2 will be explicitly distorted and invalidated if nurses were confused in rating questions based on a distinct perspective of being bullied or engaging in bullying.

\section{Limitations and future study}

The interpretation and generalization of the conclusions of this study should be carried out with caution. First, the data of this study were collected in the context of a single hospital group in Taiwan. It is worth noting that any attempt to generalize the findings of this study, especially in the prediction of workplace bullying, should be made in healthcare systems with similar social and cultural contexts.

Second, although the participants were randomly and carefully selected in a unique hospital group to represent as much different as characteristics of samples, the generalization is not as strong as that sampling from a variety of hospital groups.

Third, nurses' perceptions were investigated by selfreport data with response to such more 201 items at one moment. We cannot guarantee that all of them endorsed questionnaire with carefulness and without any cheating or guessing response.

Fourth, the 201 items of the study ten components were not included in the paper due to the space limitation. Interested readers are welcome to request the questionnaire if necessary.

The bully issue is a global problem in service-originated societies, especially among nurses in the healthcare setting $[57,58]$. Our findings that nurses' negative perceptions can be predicted by attitude as an indirect effect to negative acts are required to further prove and to induce other researches in future. For instance, in a therapeutic or care process, patients with schizophrenia, caregivers practice body restraint for protecting the individual or the community, and to facilitate transportation to health facilities might be bullying behaviors if no compassion and love exist in healthcare.

Reviewing Olweus [54] identification of bullying behaviors with three prominent characteristics: negative actions, repetition, and power imbalance. It is interesting to hypothesize that when patients with schizophrenia were treated with body restraint (negative actions) frequently (repetition) by nurse authority to patients (power imbalance), giving patient family members hold negative attitudes (or thoughts) toward nurse treatments.
Comorbid mental disorders and negative perceptions will be caused, such as depression and substance drug abuse. Patient restraint will be considered as a kind of bullying behavior (i.e. negative acts).

\section{Conclusions}

This study contributes to the academic literature by applying both Rasch analysis and PLS-SEM to explore the relationships among nurses' attitudes, negative perceptions, and negative acts regarding workplace bullying under the framework of the TPB, which provides hospital nurse management with important implications for prevention of bullying, particularly to them who are tasked with providing safer and more productive workplaces to hospital nurses. Awareness of workplace bullying was recommended to other kinds of workplaces for further studies in future. Researchers and nurse superintendents should develop prevention and intervention programs directed at workplace bullying based on the perceived severity rather than only on the prevalence and frequency of bullying behaviors.

\section{Additional file}

Additional file 1. Questions illustrated for the 10 study scales.

\section{Abbreviations}

ATQ: Automatic Thoughts Questionnaires; CP-TPB: counterproductively negative effects on theory of planned behavior; CTT: classic test theory; LSAS: Liebowitz Social Anxiety Scale; SAD: social anxiety disorder; SD: standard deviations; TPB: theory of planned behavior.

\section{Authors' contributions}

SCM developed the study concept and design. TWC and SCM analyzed and interpreted the data. HHW monitored the process of this study and help responded to the reviewers' advises and comments. TWC drafted the manuscript, and all authors provided critical revisions for important intellectual content. The study was supervised by TWC. All authors read and approved the final manuscript.

\section{Author details \\ ${ }^{1}$ College of Nursing, Kaohsiung Medical University, Kaohsiung, Taiwan. ${ }^{2}$ Nursing Department, Chi-Mei Medical Center, Tainan, Taiwan. ${ }^{3}$ Research Department, Chi-Mei Medical Center, 901 Chung Hwa Road, Yung Kung Dist., Tainan 710, Taiwan. ${ }^{4}$ Department of Hospital and Health Care Administra- tion, Chia-Nan University of Pharmacy and Science, Tainan, Taiwan. ${ }^{5}$ Bachelor Program of Senior Services, Southern Taiwan University of Science and Tech- nology, Tainan, Taiwan.}

\section{Competing interests}

The authors declare that they have no competing interests.

Availability of data and materials

These research results are available in tables and Additional file 1.

\section{Consent for publication}

Not applicable.

\section{Ethics approval and consent to participate}

This study was approved and monitored by the Research Ethics Review Board of the Chi-Mei Medical Center. Demographic data were anonymously 
collected: gender, work tenure in hospitals of all types, age, marital status, and education level

\section{Funding}

There are no sources of funding to be declared.

\section{Publisher's Note}

Springer Nature remains neutral with regard to jurisdictional claims in published maps and institutional affiliations.

Received: 27 June 2017 Accepted: 1 September 2017 Published online: 15 September 2017

\section{References}

1. Rayner C, Keashley L. Bullying at work: a perspective from Britain and North America. In: Fox S, Spector PE, editors. Counterproductive work behavior: investigations of actors and targets. Washington, DC: American Psychological Association; 2005. p. 271-96.

2. Einarsen $\mathrm{S}$, Hoel H, Notelaers $\mathrm{G}$. Measuring exposure to bullying and harassment at work: validity, factor structure and psychometric properties of the negative acts questionnaire-revised. Work Stress. 2009;23(1):24-44.

3. Leymann $\mathrm{H}$. The content and development of mobbing at work. Eur J Work Organ Psychol. 1996;5(2):165-84.

4. Chen LM, Liu KS, Cheng YY. Validation of the perceived school bullying severity scale. Educ Psychol. 2012;32(2):169-82

5. Rayner C, Cooper CL. Workplace bullying. In: Kelloway E, Barling J, Hurrell Jr J, editors. Handbook of workplace violence. Thousand Oaks: Sage; 2006. p. 47-90.

6. Zapf D, Einarsen $\mathrm{S}$, Hoel H, Vartia M. Empirical findings on bullying in the workplace. In: Einarsen S, Hoel H, Zapf D, Cooper CL, editors. Bullying and emotional abuse in the workplace: international perspectives in research and practice. New York: Taylor \& Francis; 2003.

7. Mikkelsen EG, Einarsen S. Bullying in Danish work-life: prevalence and health correlates. Eur J Work Organ Psychol. 2001;10(4):393-413.

8. Ramsay S, Troth A, Branch S. Work-place bullying: a group processes framework. J Occup Organ Psychol. 2010;84(4):799-816.

9. Tsuno K, Kawakami N, Inoue A, Abe K. Measuring workplace bullying: reliability and validity of the Japanese version of the negative acts questionnaire. J Occup Health. 2010;52(4):216-26.

10. Hutchinson M, Wilkes $L$, Jackson D, Vickers MH. Integrating individual, work group and organizational factors: testing a multidimensional model of bullying in the nursing workplace. J Nurs Manag. 2010;18(2):173-81.

11. Ma SC, Chien TW, Wang HH, Li YC, Yui MS. Applying computerized adaptive testing (CAT) to the negative acts questionnaire-revised (NAQ-R) the Rasch analysis. J Med Internet Res. 2013;16(2):e50.

12. Ajzen I. The theory of planned behavior. Organ Behav Hum Decis Process. 1991;50(2):179-211

13. Hair JF, Hult GTM, Ringle CM, Sarstedt M. A primer on partial least squares structural equation modeling (PLS-SEM). Thousand Oaks: Sage; 2014.

14. Ajzen I. From intentions to actions: a theory of planned behavior. In: Kuhl J, Beckmann J, editors. Action control: from cognition to behavior. Berlin: Springer; 1985. p. 11-39.

15. Yan Z, Cheng ECK. Primary teachers' attitudes, intentions and practices regarding formative assessment. Teach Teach Educ. 2015;45:128-36.

16. Ajzen I. Constructing a TpB questionnaire: conceptual and methodological considerations. http://www.unibielefeld.de/ikg/zick/ajzen\%20construction\%20a\%20tpb\%20questionnaire.pdf. Accessed 14 Mar 2015.

17. Hollon SD, Kendall PC. Cognitive self-statements in depression: development of an automatic thoughts questionnaire. Cogn Ther Res. 1980;4(4):383-95

18. lancu I, Bodner E, Joubran S, Lupinsky Y, Barenboim D. Negative and positive automatic thoughts in social anxiety disorder. Isr J Psychiatry Relat Sci. 2015;52(2):129-35

19. DeVellis RF. Scale development: theory and applications. 3rd ed. Thou sand Oaks: Sage Publications; 2012.
20. Rasch G. Probabilistic models for some intelligence and achievement test. Copenhagan: Danish Institute for Educational Research; Expanded ed. (1980). Chicago: The University of Chicago Press; 1960.

21. Linacre JM. Winsteps (Version 4.0.1) [Computer Software]. Beaverton, Oregon: Winsteps.com; 2011/9/3. Retrieved at http://www.winsteps. $\mathrm{com} /$.

22. Wright BD. A history of social science measurement. Educ Meas Issues Pract. 1997;16(4):33-45.

23. Bond TG, Fox CM. Applying the Rasch model: fundamental measurement in the human sciences. 2nd ed. Mahwah: Lawrence Erlbaum; 2007.

24. Linacre JM. A user's guide to WINSTEPS/MINISTEP: Rasch-model computer programs. Chicago: Winsteps; 2015.

25. Panayides $P$, Robinson C, Tymms P. The assessment revolution that has passed England by: Rasch measurement. Br Educ Res J. 2010;36(4):611-26.

26. Tormakangas K. Advantages of the Rasch measurement model in analyzing educational tests: an applicator's reflection. Educ Res Eval Int J Theory Pract. 2011:17(5):307-20.

27. Wright $\mathrm{BD}$, Linacre JM. Reasonable mean-square fit values. Rasch Meas Trans. 1994:8:370.

28. Linacre JM. Optimizing rating scale category effectiveness. J Appl Meas. 2002;3(1):85-106.

29. Haenlein M, Kaplan AM. A beginner's guide to partial least squares analysis. Underst Stat. 2004;3:283-97.

30. Hart DL, Wright BD. Development of an index of physical functional health status in rehabilitation. Arch Phys Med Rehabil. 2002;83(5):655-65.

31. Fisher WP Jr. Fuzzy truth and the Rasch model. Rasch Meas Trans. 1995;9(3):442.

32. Ludlow LH, O'Leary M. Scoring omitted and not-reached items: practical data analysis implications. Educ Psychol Meas. 1999;59(4):603-15.

33. Montiel-Overall P. Implications of missing data in survey research. Can J Inf Libr Sci. 2006;30(3):241-70.

34. Moulton M. One ruler, many tests: a primeron test equating. EDS Publications. http://www.eddata.com/resources/publications/EDS_APEC_Equating_Moulton.pdf. Accesssed 15 Mar 2015.

35. Peugh JL, Enders CK. Missing datain educational research: a review of reporting practices and suggestions for improvement. Rev Educ Res. 2004:74(4):525-56

36. Ringle CM, Sarstedt M, Straub DW. A critical look at the use of PLS-SEM in MIS quarterly. MIS Q. 2012;36:iii-xiv.

37. Hair JF, Sarstedt M, Ringle CM, Mena JA. An assessment of the use of partial least squares structural equation modeling in marketing research. J Acad Mark Sci. 2012;40:414-33.

38. Hair JF, Sarstedt M, Pieper TM, Ringle CM. The use of partial least squares structural equation modeling in strategic management research: a review of past practices and recommendations for future applications. Long Range Plan. 2012;45:320-40.

39. Lee L, Petter S, Fayard D, Robinson S. On the use of partial least squares path modeling in accounting research. Int J Account Inf Syst. 2011;12:305-28.

40. Sarstedt M, Ringle CM, Smith D, Reams R, Hair JF. Partial least squares structural equation modeling (PLS-SEM): a useful tool for family business researchers. J Fam Bus Strategy. 2014:5:105-15.

41. Peng DX, Lai F. Using partial least squares in operations management research: a practical guideline and summary of past research. J Oper Manag. 2012;30:467-80.

42. Sosik JJ, Kahai SS, Piovoso MJ. Silver bullet or voodoo statistics? A prime for using the partial least squares data analytic technique in group and organization research. Group Organ Manag. 2009;34:5-36.

43. Astrachana CB, Patelb VK, Wanzenriedc G. A comparative study of CB-SEM and PLS-SEM for theory development in family firm research. J Fam Bus Strategy. 2014;5(1):116-28.

44. Farrell GA, Bobrowski C, Bobrowski P. Scoping workplace aggression in nursing: findings from an Australian study. J Adv Nurs. 2006;55(6):778-87.

45. Hutchinson M, Vickers $M$, Jackson D, Wilkes L. They stand you in a corner; you are not to speak': nurses tell of abusive indoctrination in work teams dominated by bullies. Contemp Nurse. 2006;21(2):228-38.

46. Lewis M. Nurse bullying: organizational considerations in the maintenance and perpetration of health care bullying cultures. J Nurs Manag. 2006;14(1):52-8. 
47. Hallberg L, Strandmark M. Health consequences of workplace bullying: experiences from the perspective of employees in the public service sector. Int J Qual Stud Health Wellbeing. 2006;1(2):109-19.

48. Randle J. Bullying in the nursing profession. J Adv Nurs. 2003:43(4):395-401.

49. Quine L. Workplace bullying in nurses. J Health Psychol. 2001;6(1):73-84

50. Mikkelsen EG, Einarsen S. Relationships between exposure to bullying at work and psychological and psychosomatic health complaints: the role of state negative affectivity and generalized self-efficacy. Scand J Psychol. 2002;43(5):397-405.

51. Kivimakia M, Virtanen M. Workplace bullying and the risk of cardiovascular disease and depression. Occup Environ Med. 2003;60:779-83.

52. Einarsen SE, Mikkelsen EG. Individual effects of exposure to bullying at work. In: Einarsen SE, Hoel H, Zapf D, Cooper CL, editors. Bullying and emotional abuse in the workplace. International perspectives in research and practice. London: Taylor \& Francis; 2003. p. 127-44.

53. Kivimakia M, Elovainiob M, Vahterac J. Workplace bullying and sickness absence in hospital staff. Occup Environ Med. 2000:57:656-60.
54. Nielsen MB, Skogstad A, Matthiesen SB, Glasø L, Aasland MS, Notelaers $G$, et al. Prevalence of workplace bullying in Norway: comparisons across time and estimation methods. Eur J Work Organ Psychol. 2009;18(1):81-101.

55. Craig W, Harel-Fisch Y, Fogel-Grinvald H, Dostaler S, Hetland J, SimonsMorton B, Molcho M, de Mato MG, Overpeck M, Due P, Pickett W, HBSC Violence \& Injuries Prevention Focus Group, HBSC Bullying Writing Group. A cross-national profile of bullying and victimization among adolescents in 40 countries. Int J Public Health. 2009;54(Suppl 2):216-24.

56. Olweus D. Bullying at school: what we know and what we can do. Oxford: Blackwell; 1993.

57. Karatza C, Zyga S, Tziaferi S, Prezerakos P. Workplace bullying and general health status among the nursing staff of Greek public hospitals. Ann Gen Psychiatry. 2016;15:7

58. Ma SC, Wang HH, Chien TW. A new technique to measure online bullying: online computerized adaptive testing. Ann Gen Psychiatry. 2017:16:26.

\section{Submit your next manuscript to BioMed Central and we will help you at every step:}

- We accept pre-submission inquiries

- Our selector tool helps you to find the most relevant journal

- We provide round the clock customer support

- Convenient online submission

- Thorough peer review

- Inclusion in PubMed and all major indexing services

- Maximum visibility for your research

Submit your manuscript at www.biomedcentral com/submit 